\title{
Surface Plasmon Coupling and Its Universal Size Scaling in Metal Nanostructures of Complex Geometry: Elongated Particle Pairs and Nanosphere Trimers
}

\author{
Prashant K. Jain and Mostafa A. El-Sayed* \\ Laser Dynamics Laboratory, School of Chemistry and Biochemistry, Georgia Institute of Technology, Atlanta, \\ Georgia 30332
}

Received: December 22, 2007; In Final Form: January 21, 2008

\begin{abstract}
Recently, we showed that the plasmon resonance coupling between two interacting metal nanoparticles decays with the interparticle separation (in units of particle size) with the same universal trend independent of particle size or shape, metal type, or medium. This universal scaling behavior has been shown to apply to lithographically fabricated nanoparticle pairs, the metal nanoshell, plasmonic dielectric sensors, and the plasmon ruler useful in determining intersite distances in biological systems. In this article, we use electrodynamic simulations to examine the general applicability of this universal scaling behavior to more complex nanostructure geometries, for example, head-to-tail dimers of elongated particles of different aspect ratios and curvatures and a trimer of nanospheres. We find that the plasmon coupling between two elongated nanoparticles interacting head-to-tail decays according to the same universal law if the interparticle separation is scaled by the particle long-axis dimension. The absolute plasmon coupling strength, however, depends on the particle shape (i.e., aspect ratio and curvature), without affecting the universal scaling behavior. We also show that universal scaling is valid in a system of three interacting nanospheres, a first step toward extending this model to chains/arrays/assemblies of metal nanoparticles.
\end{abstract}

\section{Introduction}

The surface plasmon resonance oscillation of electrons in noble-metal nanostructures ${ }^{1-3}$ imparts them with large field enhancements, which result in strong light scattering and absorption $^{4-6}$ that has been utilized in a variety of applications ranging from nanophotonics ${ }^{7}$ to biomedicine.$^{8-11}$ In recent years, there has been increasing interest in the coupling of these surface plasmon fields in assembled metal nanostructures. ${ }^{12-20}$ For instance, when gold nanospheres assemble, the surface plasmon resonance frequency shifts to lower energies with respect to the single-particle resonance because of the favorable coupling of the electronic oscillations of the interacting particles. ${ }^{21-23}$ This assembly-induced plasmon resonance shift has become an effective optical/spectroscopic tool for biological sensing by utilizing gold nanoparticles (conjugated with specific recognition ligands) that assemble only in the presence of the target analyte. ${ }^{21,24}$ The field coupling between the nanoparticles also results in a large field enhancement at the interparticle junction, ${ }^{25}$ which has the ability to amplify the Raman scattering from adsorbed molecules by up to 14-15 orders of magnitude. ${ }^{26-28}$

There has been an increasing interest in the distance dependence of plasmon coupling. ${ }^{13,29-32}$ Studies on metal nanoparticle pairs fabricated with systematically varying interparticle separations using electron-beam lithography have shown that the plasmon resonance of a pair of interacting particles (of a size much smaller than the wavelength of light such that there are no severe retardation effects) red shifts exponentially with decreasing interparticle separation. ${ }^{13,14,30} \mathrm{Su}$ et al. showed that, when the interparticle separation is scaled by the particle size, this exponential trend becomes independent of the particle size. ${ }^{13}$

* Corresponding author. E-mail: melsayed@gatech.edu. Tel.: (404) 894 0292. Fax: (404) 894-0294.
We found that the fractional shift $\left(\Delta \lambda / \lambda_{0}\right)$ of the plasmon resonance wavelength decays over an interparticle separation $(s)$, which is roughly 0.2 in units of the particle dimension $(D)$, for nanodisk pairs of both gold ${ }^{30}$ and silver ${ }^{14}$ studied experimentally and in simulations of gold nanosphere pairs. ${ }^{30} \mathrm{We}$ found that the scaling behavior expressed as $\Delta \lambda / \lambda_{0} \sim k \exp ^{-s / 0.2 D}$ is universal independent of the particle size or shape, the type of metal, or the medium. ${ }^{30} \mathrm{We}$ also showed that the universal scaling model gave a "plasmon ruler equation" 30 that was able to calculate, in good agreement with the known experimental values, the interparticle separation in pairs of gold nanospheres assembled by DNA linkers with varying numbers of base pairs ${ }^{32}$ from their observed plasmon shifts. Thus the model is potentially helpful for the determination of intersite distances in biological systems using the plasmon ruler developed by Alivisatos and Liphardt. ${ }^{29,32,33}$ The universal scaling model not only gives a simple intuitive picture of plasmon coupling in metal nanostructures, but is also very general. The plasmon resonance of a metal nanoshell, ${ }^{34}$ which results from the coupling between inner- and outer-shell surface plasmons, ${ }^{35}$ also follows a similar universal size-scaling behavior with respect to the metal shell thickness, in units of the core size. ${ }^{36}$ Even the dielectric sensitivity of assembled nanostructures such as a metal nanoshell gets enhanced with increasing plasmon coupling, as per the universal scaling model. ${ }^{37}$

The size-scaling behavior and its universality has been deduced from studies on nanodisk and nanosphere pairs, in which cases the choice of the particle dimension $D$ to be used for the scaling is trivial. However, the choice of the geometric dimension to be used for the size scaling of the plasmon coupling in complex assemblies, for example, a pair of elongated particles, is less clear. In this article, we use electrodynamic simulations to study the plasmon coupling in pairs of elongated particles assembled head-to-tail. We find that the distance 
TABLE 1: Different Nanostructure Geometries Simulated Using the DDA

\begin{tabular}{|c|c|c|}
\hline $\begin{array}{l}\text { Nanostructure } \\
\text { Geometry }\end{array}$ & $\begin{array}{c}\text { Particle } \\
\text { Dimensions }\end{array}$ & $\begin{array}{c}\text { Number of Discrete } \\
\text { Dipoles/Particle }\end{array}$ \\
\hline Dimer of spheroids & $\begin{array}{l}\text { Short-axis }=10 \mathrm{~nm} \\
\text { Long-axis }=20 \mathrm{~nm}\end{array}$ & 8320 \\
\hline Dimer of spheroids & $\begin{array}{l}\text { Short-axis }=10 \mathrm{~nm} \\
\text { Long-axis }=30 \mathrm{~nm}\end{array}$ & 12632 \\
\hline Dimer of rods & $\begin{array}{l}\text { Width } \sim 18 \mathrm{~nm} \\
\text { Length } \sim 72 \mathrm{~nm}\end{array}$ & 2952 \\
\hline Dimer of cylinders & $\begin{array}{l}\text { Diameter } \sim 17 \mathrm{~nm} \\
\text { Length } \sim 52 \mathrm{~nm}\end{array}$ & 2400 \\
\hline Dimer of cylinders & $\begin{array}{l}\text { Diameter } \sim 17 \mathrm{~nm} \\
\text { Length } \sim 70 \mathrm{~nm}\end{array}$ & 3200 \\
\hline Trimer of spheres & Diameter $=10 \mathrm{~nm}$ & 4224 \\
\hline
\end{tabular}

dependence of the plasmon coupling follows the same universal scaling trend as observed earlier if the interparticle distance is scaled by the long-axis dimension. The universal scaling behavior is not affected by variations in the particle curvature and aspect ratio; however, these factors determine the absolute plasmon shift resulting from the interparticle coupling. We also find that the plasmon shift in a system of three interacting gold nanospheres (arranged in a chain) decays as a function of the ratio of the interparticle separation to the particle size with the same universal scaling behavior as that observed for particle pairs.

\section{Methods}

We used the discrete dipole approximation (DDA) method $^{38}$ to simulate the extinction efficiency $\left(Q_{\text {ext }}\right)$ spectra of nanoparticle pairs. In principle, in the DDA procedure, the target geometry is discretized into several virtual dipoles. The interaction of the light with each dipole is solved taking into account the interactions between dipoles. This simple procedure makes the DDA a powerful method for simulating the electrodynamics of nanostructures of any arbitrary geometry. ${ }^{4,39}$ In the DDA, each nanoparticle is represented by several thousand dipoles, along with a radiative reaction correction. As a result, finite-size effects including higher-order oscillation modes, radiation damping, and electromagnetic retardation are taken into account, unlike in a purely dipolar model where each particle is represented by a single dipole. ${ }^{14,30}$ To achieve reliable results, the number of dipoles in the DDA is ensured to be large enough such that the discrete-dipole spacing is much smaller than the wavelength of light and the nanostructure dimensions.
The different nanostructure geometries that we simulated included a dimer of spheroids for two different aspect ratios, a dimer of cylinders for two different aspect ratios, a dimer of hemisphere-end-capped rods, and a trimer of nanospheres. For each case, the extinction efficiency $\left(Q_{\text {ext }}\right)$ spectrum was obtained as a function of the interparticle surface-to-surface separation $(s)$. The details of each target geometry, including the nanostructure dimensions and the number of dipoles used for the target definition in the simulation, are presented in Table 1. Although electron-beam lithography (EBL) is a powerful technique for performing such a systematic study experimentally, ${ }^{30,40}$ analysis of such a wide range of structures would be impractical. Moreover, extremely small separations and sharp curvatures are difficult to obtain even with the high resolution of EBL, and it is also difficult to separate out the effects of complex substrates (e.g., a quartz slide coated with chromium). ${ }^{30}$ Nevertheless, EBL-fabricated nanostructures can be effectively used to verify some of the results obtained in this simulation study.

In all structures, the dielectric function of the target material was assumed to be that of bulk gold. The medium was assumed to have a refractive index of $1.33+0 \mathrm{i}$ for water. The DDSCAT 6.1 code was employed for the electrodynamic solution. The light polarization direction was assumed to be parallel to the axis of interparticle interaction.

\section{Results and Discussion}

The study of elongated or rod-shaped particles is interesting because these particles have two modes of plasmon resonance: one due to electronic oscillation along the short axis and another 
due to oscillation along the long axis. ${ }^{41-43}$ The long-axis mode can be tuned in both resonance frequency and oscillator strength/ polarizability by simply adjusting the aspect ratio or the surface curvature of the particle. ${ }^{25,39,42,44}$ The high oscillator strength of the plasmons of elongated/sharp-tipped particles can be expected to give rise to very strong interparticle plasmon coupling. ${ }^{15,45}$ The strength of the plasmon coupling can be expected to be systematically tuned by changing the elongation and/or curvature of the particles; ${ }^{45,46}$ however, the roles of these factors needs to be understood. In addition, the distance dependence of plasmon coupling between elongated particles also requires elucidation. Systematic understanding of the roles of particle elongation, curvature, and interparticle separation in determining plasmon coupling is required for the development of substrates with large enhancements in Raman cross sections, ${ }^{47}$ as well as control over the resonance frequency of the Raman enhancement. The optimization of plasmon coupling also becomes useful in enhancing the dielectric sensitivity of plasmonic nanostructures for biosensing applications. ${ }^{37}$

Another interesting aspect of elongated particle pairs is that, depending on the relative orientation of the two particles, their long-axis plasmon modes can either bond favorably with each other (when aligned head-to-tail) or antibond with each other (when aligned side-by-side). ${ }^{15} \mathrm{We}$ are mainly interested in the strongly coupled bonding mode. Figure 1a shows the DDAsimulated plasmon resonances in a pair of gold spheroids interacting along their long axes (head-to-tail configuration). The light polarization direction was maintained parallel to the interparticle axis so that we could investigate the interparticle bonding of the long-axis modes, as a function of the interparticle separation ${ }^{48}$ between the two particles.

Distance Dependence and Universal Scaling. With decreasing interparticle separation, we see that the longitudinal plasmon resonance of the pair red shifts and also increases in strength. The red shift of the plasmon resonance is almost exponential with decreasing interparticle separation (Figure 1b). This is very similar to the observation in dimers of circular particles, either nanodisks ${ }^{30}$ or nanospheres. ${ }^{30,32}$ In nanodisk or nanosphere pairs, we have shown that, when the interparticle separation is expressed in units of particle diameter, the fractional plasmon shift decays near-exponentially according to a universal trend with $\tau \sim 0.2 .{ }^{30}$ In other words, the plasmon coupling strength decays over a distance that is roughly 0.2 times the particle dimension. The choice of the particle dimension to be used for the size scaling is trivial in the case of nanodisk or nanosphere pairs.

In the case of elongated particles, the choice of the geometric dimension to be used for similar scaling of the plasmon coupling is less clear. Figure 2a shows that, when the fractional plasmon shift $\left(\Delta \lambda / \lambda_{0}\right)$ in the spheroid pair is plotted versus the interparticle separation in units of the long-axis dimension, we obtain a decay constant ( $\tau=0.2$ ) that is similar to that seen universally in the nanosphere or nanodisk pairs independent of their size, metal, or medium. ${ }^{30}$ At the farthest interparticle separation, i.e., $50 \mathrm{~nm}$, which is 2.5 times the long-axis dimension, the plasmon resonance wavelength is the same $(595 \mathrm{~nm})$ as that for a single spheroid. We use the plasmon resonance at the farthest separation as $\lambda_{0}$ in each of the cases analyzed.

Effect of Particle Aspect Ratio. In a pair of spheroids with a higher aspect ratio of 3 , we see a similar decay constant $(\tau=$ 0.2 ) when the interparticle separation is expressed in units of the long-axis dimension, which is $30 \mathrm{~nm}$ in this case (Figure 2b). In this case too, the plasmon resonance wavelength (687 $\mathrm{nm}$ ) at an interparticle separation of $75 \mathrm{~nm}$, which is 2.5 times
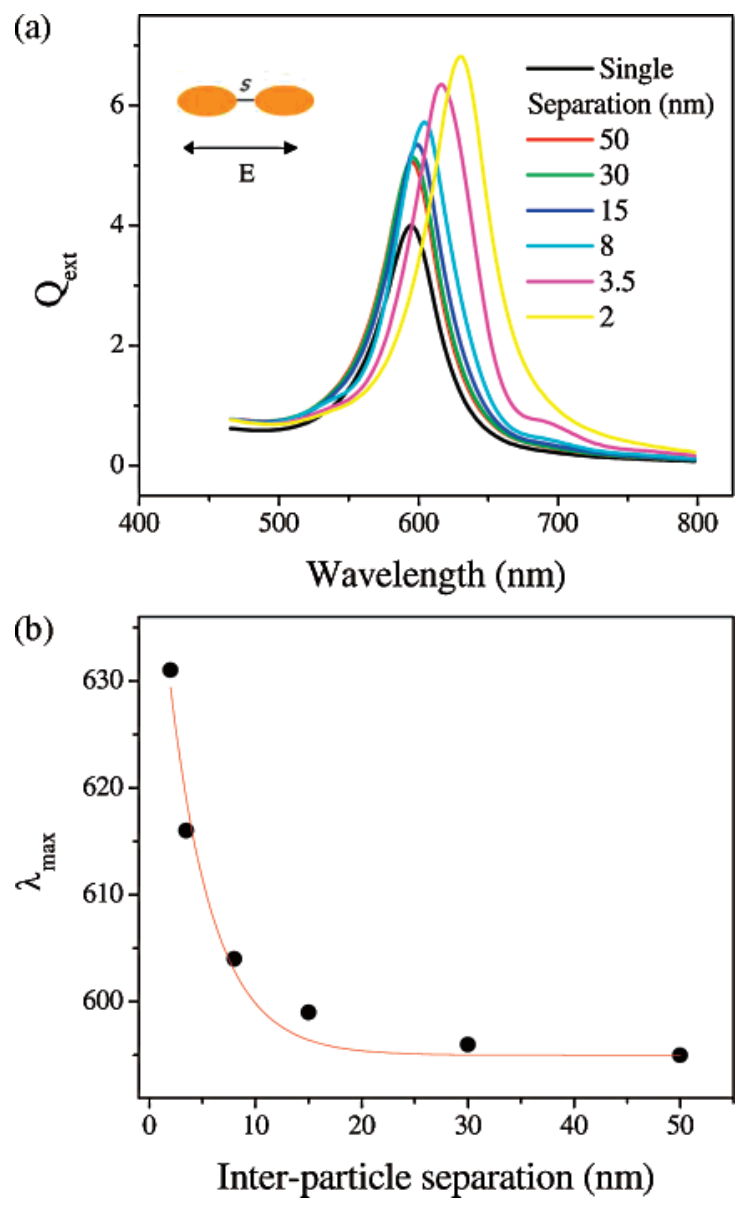

Figure 1. (a) Longitudinal plasmon resonance spectrum of a pair of gold spheroids of aspect ratio 2 interacting head-to-tail, as a function of the interparticle separation. The spectrum of a single isolated gold spheroid (short axis $=10 \mathrm{~nm}$, long axis $=20 \mathrm{~nm}$ ) is shown in black for comparison. The spectra were generated by fitting calculated data points by cubic interpolation. (b) Plasmon resonance wavelength red shifts exponentially with decreasing interparticle separation in the spheroid pair. The red line is a single-exponential decay with $R^{2}=$ 0.98063 . In the exponential-decay fit, the offset $y_{\mathrm{o}}$ was set to $595 \mathrm{~nm}$, which is the single-particle resonance wavelength.

the long-axis dimension, is nearly equal to that of the single spheroid (686 nm) (see Supporting Information). This verifies that the long-axis dimension is the appropriate dimension for the scaling of the distance dependence of the plasmon coupling.

It is also interesting to note that the fractional shifts $\left(\Delta \lambda / \lambda_{0}\right)$ for a given separation/long-axis value are quite close for the two different aspect ratios, as indicated by the similar amplitude $(k)$ values obtained from their single-exponential decay fits (Figure 2). This implies that the absolute shift $(\Delta \lambda)$ is nearly proportional to the single-particle resonance wavelength $\lambda_{0}$. The single-particle resonance wavelength for a spheroid varies directly with the aspect ratio. ${ }^{41,42}$ As a result, the absolute plasmon shift increases with increasing aspect ratio of the particle. For instance, the single spheroid of aspect ratio 2 shows a plasmon resonance wavelength maximum of $595 \mathrm{~nm}$, whereas for the spheroid of aspect ratio 3, the maximum is at $686 \mathrm{~nm}$. The absolute plasmon shift is therefore larger (for a given separation-to-long-axis ratio) in the longer-aspect-ratio spheroid pair.

Cylindrical Particle Pairs. We similarly analyzed the plasmon coupling in a pair of gold cylinders interacting headto-tail for two different aspect ratios, 3 and 4 (Figure 3a and $3 \mathrm{~b}$, respectively). Here, the aspect ratio is defined as the ratio 

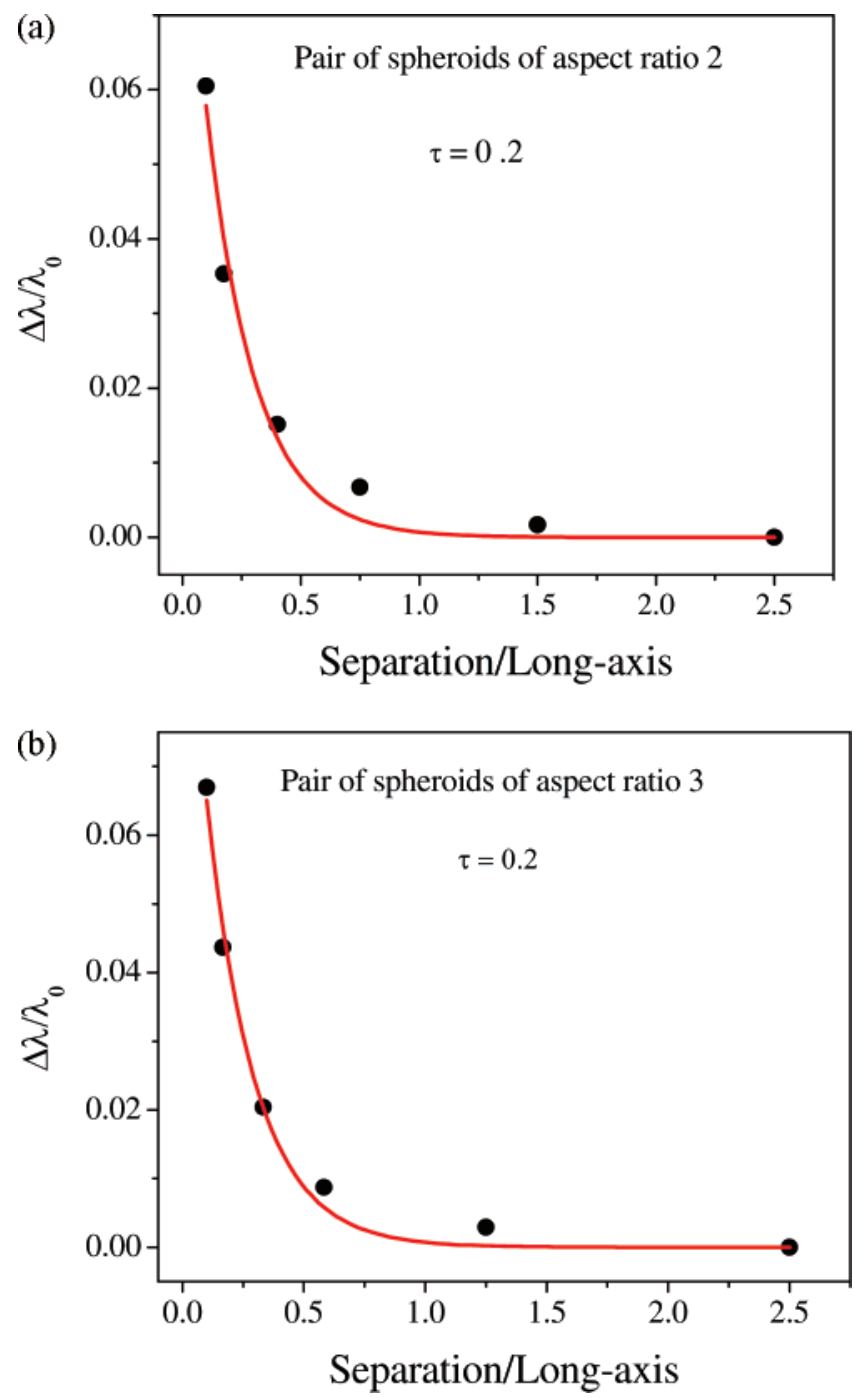

Figure 2. Fractional plasmon shift plotted as a function of the interparticle separation in units of long-axis dimension for a head-totail pair of gold spheroids with aspect ratios of (a) 2 and (b) 3 . The red curve shows the fit to the single-exponential decay $y=k \exp (-x / \tau)$, where $\tau=0.20 \pm 0.03, k=0.09 \pm 0.01$ for aspect ratio 2 and $\tau=$ $0.20 \pm 0.02, k=0.11 \pm 0.01$ for aspect ratio 3 .

of the length of the cylinder to its diameter. We see from the exponential decay fits in Figure $3 a$ and $3 b$ that the fractional plasmon shift decays over a distance roughly 0.2 times the length of the cylinder, which, in this case, is the particle dimension in the direction of coupling. Thus, the change in the particle shape from spheroidal to cylindrical does not affect the universal sizescaling behavior.

As in the case of the spheroids, it can be seen for the cylinder pairs that the amplitudes of the fits $(k)$ are very similar for the two different aspect ratios of 3 and 4 . Whereas the singlecylinder plasmon resonance is at $776 \mathrm{~nm}$ for aspect ratio 3 , it is at $904 \mathrm{~nm}$ for aspect ratio 4 (see Supporting Information). Thus, the plasmon shift at a given separation-to-length ratio in the cylinder pair is larger for the higher aspect ratio. Judging from the observations of the spheroids and cylinders, the absolute shift appears to be directly related to the elongation of the particle. On the other hand, the fractional shift and its dependence on "scaled distance" is not affected by the aspect ratio for a given shape.

Effect of Curvature. However, the fractional plasmon shift at a given separation/particle size is higher for the cylinder pair $(k=0.20)$ than for the spheroid pair $(k=0.11)$ for the same
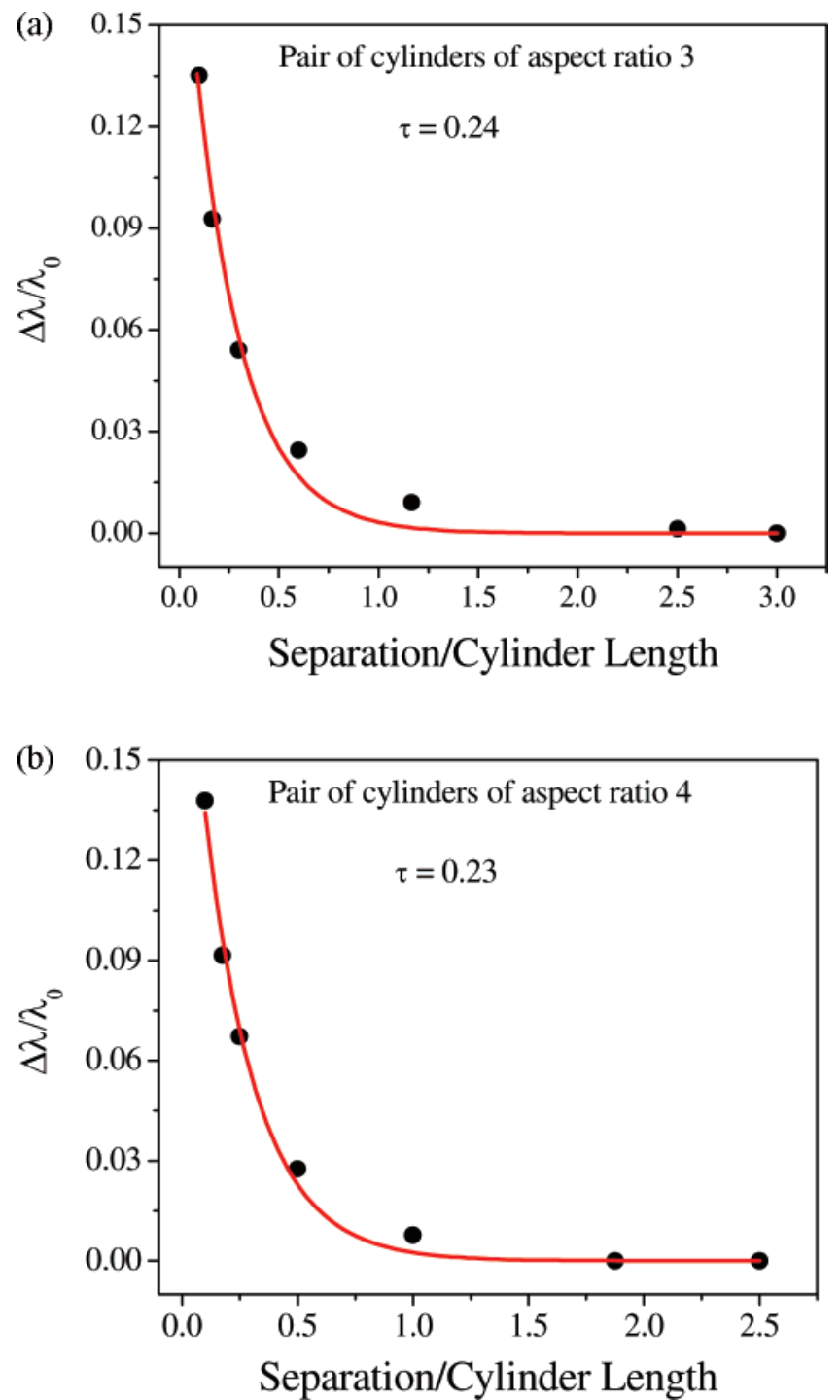

Figure 3. Fractional plasmon shift in a head-to-tail pair of gold cylinders with aspect ratios of (a) 3 and (b) 4, plotted as a function of the interparticle separation in units of the cylinder length. The red curve shows the fit to the single-exponential decay $y=k \exp (-x / \tau)$ with $\tau$ $=0.24 \pm 0.03, k=0.20 \pm 0.02$ for aspect ratio 3 and $\tau=0.23 \pm$ $0.02, k=0.21 \pm 0.01$ for aspect ratio 4 .

aspect ratio of 3 . This difference can be attributed to the abrupt end curvature (and therefore higher polarizability) of the cylinder as compared to the much smoother surface curvature of the spheroid ends. In other words, we propose that the ends of the cylinders support stronger near-fields, resulting in much stronger plasmon coupling in the cylinder pair as compared to the spheroid pair.

This explanation is further supported when we make the ends of the cylinders smoother by using hemispherical end caps (see third row in Table 1), while keeping the aspect ratio constant at 4 and the dimensions similar. The hemisphere-end-capped rod has a single-particle plasmon resonance wavelength maximum $(852 \mathrm{~nm})$ that is shorter than the single-cylinder resonance wavelength (904 nm) (see Supporting Information). At the same time, the fractional shift (and the absolute shift) in the pair of hemisphere-end-capped rods $(k=0.15)$ is considerably smaller than that in the cylinder pair $(k=0.21)$ for the same separationto-length ratio (see Figure 4). Thus, the smoother curvature of the rods as compared to the cylinders gives less strong field coupling. 


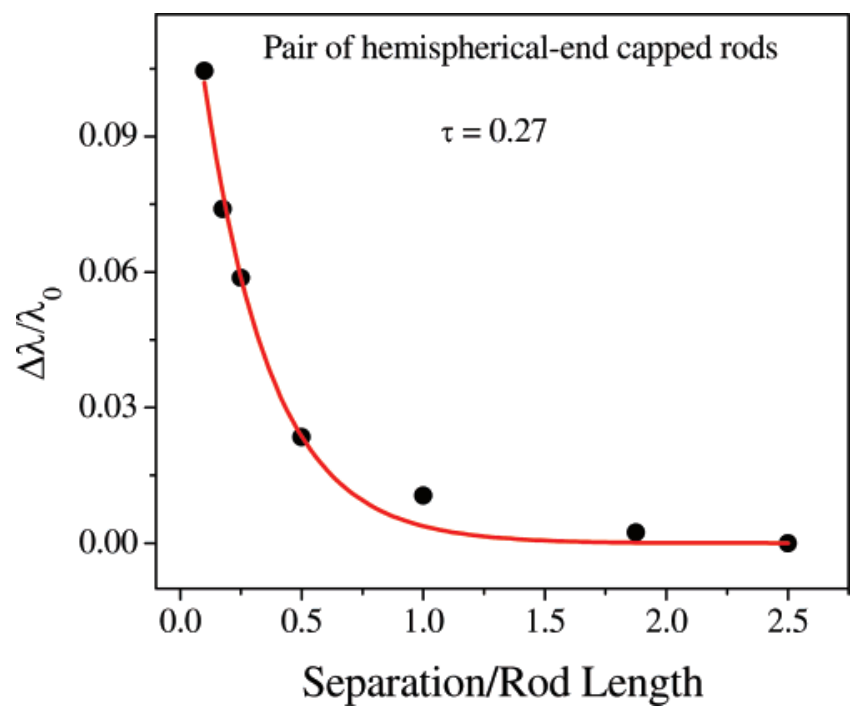

Figure 4. Fractional plasmon shift in a pair of gold hemisphere-endcapped rods of aspect ratio 4, as a function of the interparticle separation in units of the rod length. The red curve is a fit to the single-exponential decay $y=k \exp (-x / \tau)$ with $\tau=0.27 \pm 0.02, k=0.15 \pm 0.01$.
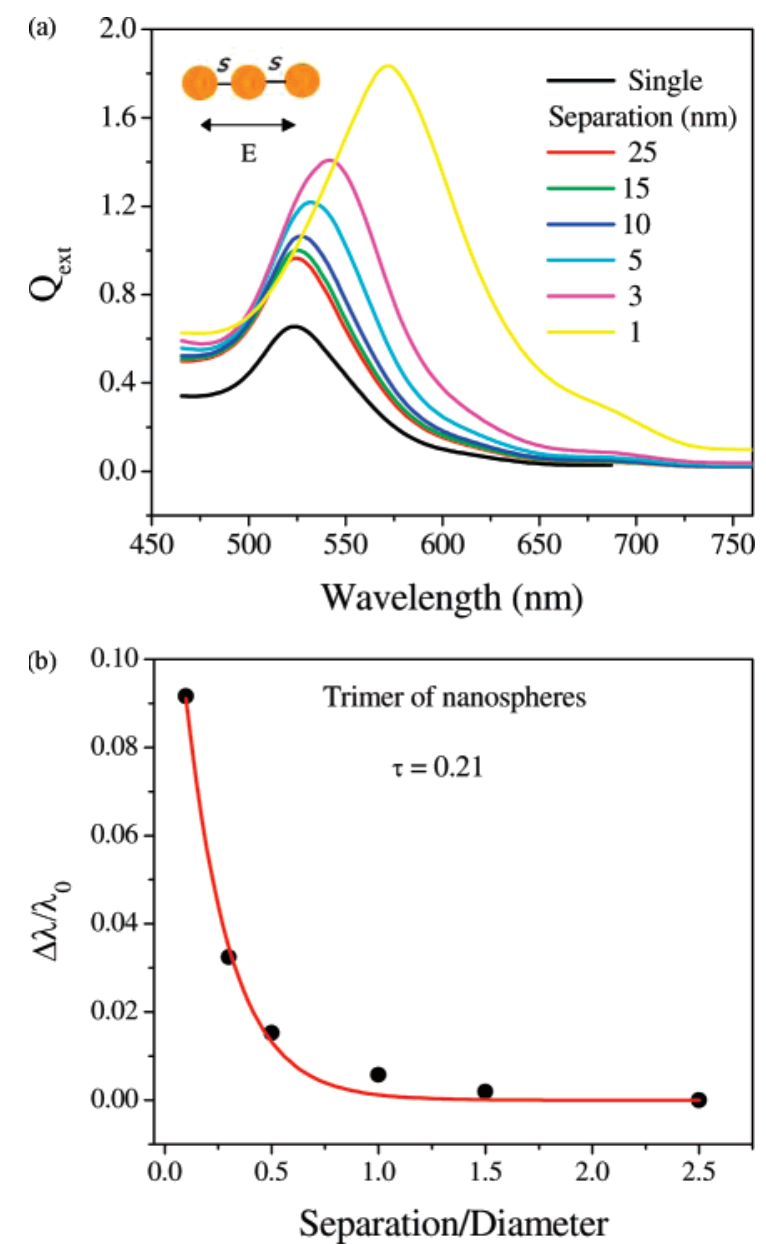

Figure 5. (a) Plasmon resonance spectrum of a "linear" trimer of gold nanospheres as a function of the interparticle separation. The spectrum of a single gold nanosphere (10-nm diameter) is shown in black for reference. (b) Fractional plasmon shift in the nanosphere trimer as a function of the interparticle separation in units of the particle diameter. The red line is a fit to the single-exponential decay $y=k \exp (-x / \tau)$ with $\tau=0.21 \pm 0.02, k=0.15 \pm 0.01$.

Extension to More than Two Particles. Figure 5a shows the simulated plasmon resonance in a trimer of gold nanospheres

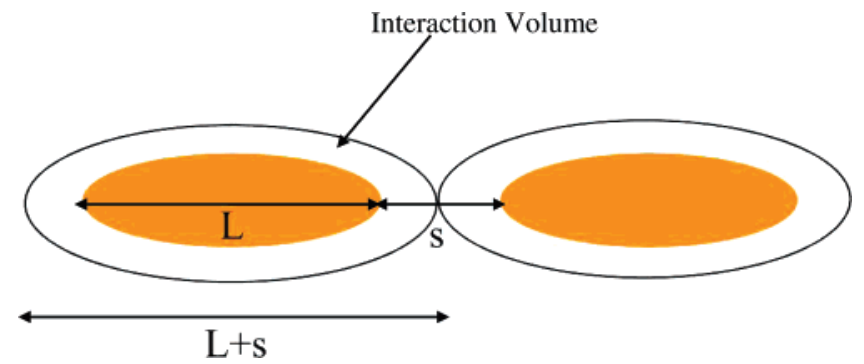

Figure 6. Depiction of an interaction volume around a metal nanospheroid (with long-axis dimension $L$ ) interacting head-to-tail with a proximal metal nanospheroid spaced by an interparticle separation $s$. The interaction volume has the same aspect ratio (i.e., shape) as the spheroid but with a dimension of $L+s$ along the direction of coupling. The ratio of the interaction volume to the particle volume is therefore given by $[(\mathrm{L}+s) / L]^{3}$.

(arranged in a linear chain) as a function of the interparticle separation between the spheres. The trimer shows a single resonance peak that red shifts with decreasing interparticle separation. Whereas the plasmon shift in the trimer is larger than that in the dimer, ${ }^{15,30}$ the fractional plasmon shift shows the same universal decay trend $(\tau=0.21)$ when the interparticle separation is scaled by the nanosphere diameter (Figure $5 b$ ).

Origin of the Universal Scaling of Plasmon Coupling. As we have explained in the past,$^{30}$ the coupling-induced plasmon shift in a metal nanoparticle pair is determined by the strength of the interparticle near-field interaction relative to the intraparticle Coulombic restoring force on the displaced (because of plasmon excitation) electron cloud. An increase in the aspect ratio and/or surface curvature of the particle results in a reduction in the intraparticle Coulombic restoring force, an increase in the single-particle polarizability (and dipole moment), and consequently a stronger near-field interaction. Therefore, the absolute plasmon shift is larger in pairs of particles with higher aspect ratios/curvatures. The distance dependence of the couplinginduced plasmon shift, however, depends only on the interparticle separation relative to the particle dimension. This is because the near-field interaction, in the dipolar quasistatic limit, decays as $1 /(\text { distance })^{3}$, whereas the intraparticle restoring force (or inverse of particle polarizability) depends on $1 /$ (particle dimension $)^{3}$. Thus, because of the inherent scaling of these two competing forces, the plasmon coupling strength varies universally as a function of the (distance/particle dimension) ratio. In the dipolar-coupling limit, the plasmon coupling strength varies as $1 /$ (distance/particle dimension $)^{3}$. However, for finite-sized particles at small interparticle separation, it is necessary to consider, in addition to the dipole-dipole interaction, coupling between modes of different orders $l$ and $l$. Such a full interaction can be represented as a sum of terms of the kind $1 /$ (distance/ particle dimension $)^{l+l^{\prime}+1}$, where $l, l^{\prime}=1,2,3$, etc., respectively, for dipole, quadrupole, octupole, etc., interactions. ${ }^{19,36}$ Analytically, this sum is reflected as an exponential decay, ${ }^{19}$ consistent with the observation in the current investigation and past studies, ${ }^{13,14,30}$ including both experiments and simulations.

The choice of the particle dimension to be used for scaling in the case of a pair of elongated particles is justified by visualizing an interaction volume around each particle, as shown in Figure 6. The ratio of the interaction volume to the actual volume of the particle gives a measure of the interparticle nearfield coupling strength relative to the intraparticle restoring force. Thus, the distance dependence of plasmon coupling in the dipolar limit is given by $1 /(s / L+1)^{3}$, where $s$ is the interparticle separation and $L$ is the particle dimension along the coupling direction. 
The concept of the scaling dimension has important implications for nanophotonics. As per the concept of the diffraction limit, two points cannot be completely resolved when they are separated by less than a distance on the order of the wavelength of light. In close analogy, on the nanoscale, two plasmonic components cannot be completely independent (i.e., they are coupled) when they are separated by less than a distance on the order of the wavelength of the plasmon mode. It is interesting to note that the particle dimension in the light polarization direction is roughly one-half the wavelength of the plasmon (dipolar) mode. Therefore, plasmon coupling decays on the order of this particle dimension universally, independent of particle size, particle shape, metal type, or medium. ${ }^{30}$

\section{Conclusion}

In summary, we have shown that the universal size-scaling behavior of plasmon coupling observed earlier in nanodisk and nanosphere pairs extends to more complex assembled plasmonic nanostructures. In the case of a pair of elongated particles assembled head-to-tail, plasmon coupling decays as a function of the interparticle separation as per the universal law if the particle dimension in the direction of the coupling is used for scaling the interparticle separation. We have also shown how the particle shape, elongation, and curvature influence the coupling-induced plasmon shift without affecting the universal size-scaling behavior. The universal scaling model is also seen to be valid in a system of three interacting particles, which is a first step in the extension of this model to larger chains/ assemblies/arrays of particles. ${ }^{22,49}$ In three-dimensional assemblies, the particle volume fraction (or its cube root) could possibly be the scaling variable corresponding to the separationto-size ratio used in the psuedo-one-dimensional cases presented here. It must be noted, however, that, as the net size of the assembled nanostructure approaches the wavelength of light, electromagnetic retardation becomes increasingly important; ${ }^{50}$ the effect of this factor on the plasmon coupling distance dependence and its universal scaling is yet to be characterized. We have not considered far-field diffractive coupling effects ${ }^{20,51,52}$ that become important at separations on the order of the light wavelength. For the interparticle separations that we have studied, near-field coupling is dominant. Our work is a step toward the detailed characterization of the distancedependent plasmon resonances in complex assemblies, which is potentially useful in the plasmon ruler application, surfaceenhanced Raman spectroscopy, and nanoparticle assembly-based biosensing. ${ }^{53}$

Acknowledgment. We thank the National Science Foundation Division of Materials Research for financial support (Grant 0527297). We thank B. T. Draine and P. J. Flatau for use of DDSCAT 6.1. Computations were supported by the Center for Computational Molecular Science and Technology at Georgia Tech, which is partially funded through a Shared University Research grant from IBM and Georgia Tech.

Supporting Information Available: Plasmon resonance as a function of interparticle separation for a pair of spheroids of aspect ratio 3 (Figure S1), a pair of gold cylinders of aspect ratio 3 (Figure S2), a pair of gold cylinders of aspect ratio 4 (Figure S3), and a pair of gold hemisphere-end-capped rods of aspect ratio 4 (Figure S4). This material is available free of charge on the Internet at http://pubs.acs.org.

\section{References and Notes}

(1) Kreibig, U.; Vollmer, M. Optical Properties of Metal Clusters; Springer: Berlin, 1995; Vol. 25.
(2) El-Sayed, M. A. Acc. Chem. Res. 2001, 34, 257.

(3) Bohren, C. F.; Huffman, D. R. Absorption and Scattering of Light by Small Particles; Wiley: New York, 1983.

(4) Kelly, K. L.; Coronado, E.; Zhao, L. L.; Schatz, G. C. J. Phys. Chem. B 2003, 107, 668.

(5) Jain, P. K.; Lee, K. S.; El-Sayed, I. H.; El-Sayed, M. A. J. Phys Chem. B 2006, 110, 7238.

(6) Jain, P. K.; Huang, X.; El-Sayed, I. H.; El-Sayed, M. A. Plasmonics 2007, 2, 107.

(7) Maier, S. A.; Brongersma, M. L.; Kik, P. G.; Meltzer, S.; Requicha, A. A. G.; Atwater, H. A. Adv. Mater. 2001, 13, 1501.

(8) Huang, X.; El-Sayed, I. H.; Qian, W.; El-Sayed, M. A. J. Am. Chem. Soc. 2006, 128, 2115.

(9) Huang, X.; Jain, P. K.; El-Sayed, I. H.; El-Sayed, M. A. Photochem. Photobiol. 2006, 82, 412.

(10) Jain, P. K.; El-Sayed, I. H.; El-Sayed, M. A. Nano Today 2007, 2, 18.

(11) Loo, C., A.; Lowery, A.; Halas, N.; West, J.; Drezek, R. Nano Lett. 2005, 5, 709 .

(12) Maier, S. A.; Brongersma, M. L.; Kik, P. G.; Atwater, H. A. Phys. Rev. B 2002, 65, 193408/1.

(13) Su, K. H.; Wei, Q.-H.; Zhang, X.; Mock, J. J.; Smith, D. R.; Schultz, S. Nano Lett. 2003, 3, 1087.

(14) Gunnarsson, L.; Rindzevicius, T.; Prikulis, J.; Kasemo, B.; Käll, M.; Zou, S.; Schatz, G. C. J. Phys. Chem. B 2005, 109, 1079.

(15) Jain, P. K.; Eustis, S.; El-Sayed, M. A. J. Phys. Chem. B 2006, 110,18243 .

(16) Sundaramurthy, A.; Crozier, K. B.; Kino, G. S.; Fromm, D. P.; Schuck, P. J.; Moerner, W. E. Phys. Rev. B 2005, 72, 165409/1.

(17) Sweatlock, L. A.; Maier, S. A.; Atwater, H. A.; Penninkhof, J. J.; Polman, A. Phys. Rev. B 2005, 71, 235408/1.

(18) Thomas, K. G.; Barazzouk, S.; Ipe, B. I.; Joseph, S. T. S.; Kamat, P. V. J. Phys. Chem. B 2004, 108, 13066.

(19) Nordlander, P.; Oubre, C.; Prodan, E.; Li, K.; Stockman, M. I. Nano Lett. 2004, 4, 899.

(20) Fromm, D. P.; Sundaramurthy, A.; Schuck, P. J.; Kino, G.; Moerner, W. E. Nano Lett. 2004, 4, 957.

(21) Elghanian, R.; Storhoff, J. J.; Mucic, R. C.; Letsinger, R. L.; Mirkin, C. A. Science 1997, 277, 1078

(22) Storhoff, J. J.; Lazarides, A. A.; Mucic, R. C.; Mirkin, C. A.; Letsinger, R. L.; Schatz, G. C. J. Am. Chem. Soc. 2000, 122, 4640.

(23) Jain, P. K.; Qian, W.; El-Sayed, M. A. J. Phys. Chem. B 2006, $110,136$.

(24) Mirkin, C. A. Inorg. Chem. 2000, 39, 2258.

(25) Hao, E.; Schatz George, C. J. Chem. Phys. 2004, 120, 357.

(26) Nie, S.; Emory, S. R. Science 1997, 275, 1102.

(27) Kneipp, K.; Wang, Y.; Kneipp, H.; Itzkan, I.; Dasari, R. R.; Feld, M. S. Phys. Rev. Lett. 1996, 76, 2444.

(28) Michaels, A. M.; Jiang, J.; Brus, L. J. Phys. Chem. B 2000, 104, 11965 .

(29) Sonnichsen, C.; Reinhard, B. M.; Liphardt, J.; Alivisatos, A. P. Nat. Biotechnol. 2005, 23, 741.

(30) Jain, P. K.; Huang, W.; El-Sayed, M. A. Nano Lett. 2007, 7, 2080.

(31) Rechberger, W.; Hohenau, A.; Leitner, A.; Krenn, J. R.; Lamprecht, B.; Aussenegg, F. R. Opt. Commun. 2003, 220, 137.

(32) Reinhard, B. M.; M. Siu; Agarwal, H.; Alivisatos, A. P.; Liphardt, J. Nano Lett. 2005, 5, 2246.

(33) Reinhard, B.; Sheikholeslami, S.; Mastroianni, A.; Alivisatos, A. P.; Liphardt, J. Proc. Natl. Acad. Sci. U.S.A. 2007, 104, 2667.

(34) Oldenburg, S. J.; Averitt, R. D.; Westcott, S. L.; Halas, N. J. Chem. Phys. Lett. 1998, 28, 243.

(35) Prodan, E.; Radloff, C.; Halas, N. J.; Nordlander, P. Science 2003, $302,419$.

(36) Jain, P. K.; El-Sayed, M. A. Nano Lett. 2007, 7, 2854.

(37) Jain, P. K.; El-Sayed, M. A. J. Phys. Chem. C 2007, 111, 17451.

(38) Draine, B. T.; Flatau, P. J. J. Opt. Soc. Am. A 1994, 11, 1491.

(39) Lee, K.-S.; El-Sayed, M. A. J. Phys. Chem. B 2005, 109, 20331.

(40) Huang, W.; Qian, W.; Jain, P. K.; El-Sayed, M. A. Nano Lett. 2007, 7,3227 .

(41) Gans, R. Ann. Phys. 1915, 47, 270.

(42) Link, S.; Mohamed, M. B.; El-Sayed, M. A. J. Phys. Chem. B 1999, 103, 3073. Erratum: J. Phys. Chem. B 2005, 109, 10531.

(43) Murphy, C. J.; Sau, T. K.; Gole, A. M.; Orendorff, C. J.; Gao, J.; Gou, L.; Hunyadi, S. E.; Li, T. J. Phys. Chem. B 2005, 109, 13857.

(44) Wiley, B. J.; Chen, Y.; McLellan, J. M.; Xiong, Y.; Li, Z.-Y.; Ginger, D.; Xia, Y. Nano Lett. 2007, 7, 1032. 
(45) Aizpurua, J.; Bryant, G. W.; Richter, L. J.; Garcia de Abajo, F. J.; Kelley, B. K.; Mallouk, T. Phys. Rev. B 2005, 71, 235420/1.

(46) Onuta, T.-D.; Waegele, M.; DuFort, C. C.; Schaich, W. L.; Dragnea, B. Nano Lett. 2007, 3, 557.

(47) Nikoobakht, B.; El-Sayed, M. A. J. Phys. Chem. A 2003, 107, 3372

(48) Note that, in all of our analyses, we used a lower bound of 0.1 on the separation-to-size ratio for consistency with current and previous studies. (49) Wei, Q.-H.; Su, K.-H.; Durant, S.; Zhang, X. Nano Lett. 2004, 4, 1067.
(50) Dahmen, C.; Schmidt, B.; von Plessen, G. Nano Lett. 2007, 7, 318

(51) Lamprecht, B.; Schider, G.; Lechner, R. T.; Ditlbacher, H.; Krenn, J. R.; Leitner, A.; Aussenegg, F. R. Phys. Rev. Lett. 2000, 84, 4721.

(52) Haynes, C. L.; McFarland, A. D.; Zhao, L.; Van Duyne, R. P.; Schatz, G. C.; Gunnarsson, L.; Prikulis, J.; Kasemo, B.; Käll, M. J. Phys. Chem. B 2003, 107, 7337.

(53) Sudeep, P. K.; Joseph, S. T. S.; Thomas, K. G. J. Am. Chem. Soc. 2005, 127, 6516. 